\title{
Fatores de Risco para Infecções de Sítio Cirúrgico em Pacientes Operadas por Câncer de Mama
}

\author{
Risk factors for Wound Infection in Operated Breast Cancer Patients \\ Hermes de Freitas Barbosa, Francisco José Candido dos Reis, \\ Hélio Humberto Angotti Carrara, Jurandyr Moreira de Andrade
}

\begin{abstract}
RESUMO
Objetivo: identificar os fatores de risco associados à ocorrência de infecção de sítio cirúrgico (ISC) em cirurgias para tratamento do câncer de mama.

Métodos: foram incluidas 140 pacientes submetidas à cirurgia para tratamento do câncer invasor de mama, no periodo de janeiro de 2001 a dezembro de 2002. A infecção do sítio cirúrgico foi definida como aquela que ocorreu em até 30 dias após a cirurgia e esteve relacionada à mesma, segundo critérios-padrão adotados pelo CDC (Centers for Disease Control and Prevention - USA). As ISC foram consideradas superficiais quando envolviam somente pele e tecido subcutâneo, e profundas quando envolviam tecidos profundos da incisão, como fáscias e musculatura. Foram estudados fatores de risco associados às pacientes (idade, status hormonal, estadiamento, indice de massa corporal (IMC) e hemoglobina). Os fatores relacionados à cirurgia foram: tipo, tempo de internação, duração e formação de seroma e hematoma. A análise estatística foi feita com o teste de Mann-Whitney (variáveis numéricas não paramétricas) e teste exato de Fisher para variáveis quantitativas.

Resultados: do total de 140 pacientes incluidas, 29 (20,7\%) apresentaram ISC, sendo 19 $(13,6 \%)$ superficiais e 10 (7,1\%) profundas; 111 pacientes não apresentaram ISC, sendo consideradas como grupo controle. Os fatores de risco associados à paciente e à doença foram o estádio localmente avançado (odds ratio $=2,7$; IC 95\%: 1,1-6,5) e a obesidade representada pelo IMC médio de $32,2 \mathrm{~kg} / \mathrm{m}^{2}$ nas pacientes com ISC e 27,2 $\mathrm{kg} / \mathrm{m}^{2}$ no grupo controle $(p<0,0001)$. Os fatores ligados ao tratamento da doença foram o uso de quimioterapia neoadjuvante com odds ratio de 2,7 (IC 95\%: 1,1-6,5), a duração da cirurgia, que apresentou mediana de 165 minutos nas pacientes que desenvolveram a infecção e de 137 minutos no grupo controle $(p=0,02)$, e o número de dias de utilização de dreno no pós-operatório, que teve mediana de 6 entre as pacientes com ISC e 5 no grupo controle ( $p=0,048)$.

Conclusão: a partir da identificação de fatores de risco como o estádio avançado, a realização de quimioterapia neoadjuvante e a obesidade, pode-se reforçar a necessidade de cuidados pré-operatórios com estas pacientes. A utilização de técnica cirúrgica acurada pode reduzir o impacto dos outros fatores, ou seja, o tempo cirúrgico e o tempo de dreno.
\end{abstract}

PALAVRAS-CHAVE: Infecções cirúrgicas. Mama: câncer. Cirurgia: complicações. Obesidade. Diabete melito.

Departamento de Ginecologia e Obstetrícia da Faculdade de Medicina de Ribeirão Preto - Universidade de São Paulo Correspondência:

Francisco José Candido dos Reis

Departamento de Ginecologia e Obstetrícia

Av. Bandeirantes $3900,8^{\circ}$ andar

14049-900 - Ribeirão Preto - SP

Telefone: (16) 602-2589 - Fax: (16) 633-0946

e-mail: fjcreis@fmrp.usp.br

\section{Introdução}

A cirurgia é método de grande importância no tratamento da paciente com câncer da mama, tendo papel fundamental no controle loco-regional da doença e na definição de parâmetros para a indicação de tratamento adjuvante. Diversos fa- 
tores têm contribuído para a segurança dos procedimentos cirúrgicos na atualidade, entre eles a consagração de técnicas mais conservadoras, o aprimoramento da anestesia e a utilização de antibioticoprofilaxia. No entanto, a cirurgia para o câncer de mama pode ser acompanhada de complicações, com impacto na qualidade do tratamento e nos custos associados.

A infecção do sítio cirúrgico (ISC) permanece como uma das causas mais comuns de morbimortalidade no paciente cirúrgico, apesar dos avanços na prática cirúrgica e o uso difundido de antibióticos profiláticos. Cada episódio de ISC aumenta a duração da internação em aproximadamente sete dias e conseqüentemente os custos hospitalares ${ }^{1-3}$. Os custos com o tratamento após a alta hospitalar também são maiores, uma vez que a paciente necessitará de medicações, adiará sua volta ao trabalho e sua qualidade de vida piorará. A presença de uma ISC na paciente com câncer de mama adia o início da terapia adjuvante e há evidências que sugerem que os resultados para a paciente em termos de controle local e sobrevida estarão comprometidos ${ }^{4}$.

A maioria dos estudos sugere que a taxa de infecção em cirurgia limpa é de $5 \%{ }^{5}$. Entretanto, quando os pacientes são seguidos intensivamente por seis semanas após a cirurgia e a definição de infecção não for limitada apenas à presença de secreção purulenta, esta taxa pode chegar a $10 \%$.

A prevenção da ISC demanda conhecimento preciso dos fatores de risco para a sua ocorrência. Dentre estes fatores de risco, alguns estudos destacam a idade avançada ${ }^{6,7}$, a obesidade ${ }^{6}$ e a formação de seroma ${ }^{8}$.

O objetivo deste trabalho foi identificar fatores de risco para o desenvolvimento de infecção de sítio cirúrgico em cirurgias para câncer de mama.

\section{Pacientes e Métodos}

No período de janeiro de 2001 a dezembro de 2002, foram realizadas 142 cirurgias para tratamento do câncer invasor de mama. Duas pacientes eram procedentes de localidades distantes, com dificuldade de transporte, motivo pelo qual tiveram o pós-operatório acompanhado em outro serviço, e não foram incluídas no estudo por não possuirem registros fidedignos do seguimento pósoperatório. O projeto foi aprovado pela Comissão de Ética em Pesquisa do Hospital das Clínicas de Ribeirão Preto.
Foi realizado estudo observacional tipo casocontrole incluindo 140 pacientes submetidas à cirurgia para tratamento do câncer de mama. Todas as pacientes foram submetidas a antibioticoprofilaxia com cefazolina $2 \mathrm{~g}$ endovenosa, 30 minutos antes da cirurgia, com exceção de cinco casos de pacientes com indicação de profilaxia para endocardite bacteriana, nas quais foram utilizadas gentamicina e ampicilina.

A ISC foi definida como aquela diagnosticada pela equipe médica, que ocorreu em até 30 dias após a cirurgia e esteve relacionada à mesma. Esta definição está de acordo com os critérios-padrão adotados pelo CDC (Centers for Disease Control and Prevention - USA $)^{9}$. As ISC foram consideradas superficiais quando envolviam somente pele e tecido subcutâneo, e profundas quando envolviam tecidos profundos da incisão, como musculatura e fáscias.

Foram considerados casos as pacientes que apresentaram ISC após o tratamento cirúrgico do câncer de mama e controles aquelas pacientes que evoluíram sem ISC segundo os padrões adotados pelo CDC. Não foram incluídas as pacientes submetidas a cirurgia para tratamento do carcinoma in situ e de condições benignas da mama e as pacientes submetidas apenas a procedimentos diagnósticos, ou seja, biópsias incisionais ou excisionais.

Os fatores de risco relacionados à paciente foram: a idade em anos, o status hormonal (menacme ou menopausa), a presença de diabete mellito, o estadiamento (segundo a FIGO) do câncer de mama no momento do diagnóstico, o índice de massa corporal e o valor da hemoglobina préoperatória em g/dL.

Os fatores de risco relacionados ao tratamento do câncer de mama foram: o tipo de cirurgia realizada, o tempo de internação prévia em dias, a duração da cirurgia em minutos, a formação ou não de seroma, a formação ou não de hematoma, o tempo de permanência do dreno em dias, e o uso de quimioterapia neoadjuvante. Foram consideradas cirurgias conservadoras a tumorectomia com linfadenectomia axilar e a quadrantectomia com linfadenectomia axilar; a mastectomia com linfadenectomia axilar foi considerada cirurgia radical.

Os dados foram obtidos da análise dos prontuários médicos das pacientes e inseridos em banco de dados do programa Microsoft Access 2002. A análise estatística foi realizada com auxílio do software GraphPad Prism versão 3.00. Foram utilizados o teste de Mann-Whitney, para análise de variáveis numéricas não paramétricas, o teste exato de Fisher, para análise de variáveis qualitativas, e o teste $t$, para análise de variáveis quantitativas contínuas não pareadas. 


\section{Resultados}

Do total de 140 pacientes incluídas, 29 $(20,7 \%)$ apresentaram ISC, sendo $19(13,6 \%)$ superficiais e $10(7,1 \%)$ profundas, e 111 pacientes não apresentaram ISC, sendo consideradas como grupo controle.

$\mathrm{Na}$ avaliação dos fatores de risco, verificamos que entre as pacientes com ISC a média de idade foi 56,9 anos (31-81) e entre as pacientes controles, 55, 5 anos $(25-86)(p=0,31$; teste $t)$. Entre as pacientes com ISC, $19(65,5 \%)$ estavam na pós-menopausa e no grupo controle, 71 (64\%) ( $p=0,53$; teste exato de Fisher). Entre as pacientes que desenvolveram ISC, $6(20,7 \%)$ tinham diabete melito e no grupo controle, $13(11,7 \%)$ (odds ratio $=1,97$; IC 95\%: 0,67-5,73, $\mathrm{p}=0,17$; teste exato de Fisher).

Outro fator analisado foi o estadiamento inicial; observou-se que entre as pacientes que desenvolveram ISC, $20(69,0 \%)$ apresentavam estádio localmente avançado (IIB, IIIA, IIIB e IV) contra $50(45,0 \%)$ do grupo controle (odds ratio $=$ 2,7; IC 95\%: 1,1-6,5, $\mathrm{p}=0,02$; teste exato de Fisher). A média do índice de massa corporal nas pacientes com ISC foi $32,2 \mathrm{~kg} / \mathrm{m}^{2}(20,5-50,3) \mathrm{e}$ nas pacientes controles, $27,2 \mathrm{~kg} / \mathrm{m}^{2}(17,1-47,4)$ $(\mathrm{p}<0,0001$, teste $t)$. A hemoglobina pré-operatória foi de $12,7 \mathrm{~g} / \mathrm{dL}(10,5-14,9)$ nas pacientes com ISC e $12,9 \mathrm{~g} / \mathrm{dL}(9,1-16,4)$ nas controles $(\mathrm{p}=0,26$; teste $t$ ) (Tabela 1 ).

Tabela 1 - Fatores de risco para a ocorrência de infecção do sítio cirúrgico associados a condições da paciente e à doença.

\begin{tabular}{|c|c|c|c|}
\hline & ISC & Controles & $p$ \\
\hline Idade (anos) & $56,9(31-81)$ & $55,5(25-86)$ & ns \\
\hline Gestações & $4(0-12)$ & $3(0-21)$ & ns \\
\hline Pós-menopausa & $19(65,5 \%)$ & $71(64,0 \%)$ & ns \\
\hline Diabete Melito & $6(20,7 \%)$ & $13(11,7 \%)$ & ns \\
\hline \multicolumn{4}{|l|}{ Estádio localmente } \\
\hline avançado & $20(68,9 \%)$ & $50(45,0 \%)$ & 0,02 \\
\hline Estádio I & $2(6,9 \%)$ & $28(25,2 \%)$ & \\
\hline Estádio IIA & $7(24,1 \%)$ & $33(29,8 \%)$ & \\
\hline Estádio IIB & $6(20,7 \%)$ & $18(16,2 \%)$ & \\
\hline Estádio IIIA & $1(3,4 \%)$ & $8(7,2 \%)$ & \\
\hline Estádio IIIB & $11(37,9 \%)$ & $20(18,0 \%)$ & \\
\hline Estádio IV & $2(6,9 \%)$ & $4(3,6 \%)$ & \\
\hline IMC $\left(\mathrm{kg} / \mathrm{m}^{2}\right)$ & $32,2(20,5-50,3)$ & $27,2(17,1-47,4)$ & $<0,0001$ \\
\hline \multicolumn{4}{|l|}{ Hemoglobina } \\
\hline (pré-operatória)(g/dL) & $12,7(10,5-14,9)$ & $12,9(9,1-16,4)$ & ns \\
\hline
\end{tabular}

$\overline{I S C}=$ infecção de sítio cirúrgico; IMC = índice de massa corporal; ns = não significante.
As pacientes que receberam quimioterapia neoadjuvante apresentaram risco significativamente maior para a ocorrência de ISC, com odds ratio de 2,7 (IC 95\%: 1,1-6,5). Entre as pacientes com ISC, $20(69,0 \%)$ foram tratadas com quimioterapia neoadjuvante e no grupo controle, $50(45,1 \%)(p=0,02$; teste exato de Fisher). O período de internação prévia apresentou mediana de um dia (0-6) tanto entre as pacientes com ISC quanto entre as pacientes controles $(0-9)(p=0,36$; teste de Mann-Whitney). Outro fator que elevou de forma significante o risco de ISC foi a duração da cirurgia, que apresentou mediana de $165 \mathrm{mi}-$ nutos (80-340) nas pacientes que desenvolveram a infecção e de 137 minutos (45-350) nas pacientes controles ( $p=0,02$; teste de Mann-Whitney). Entre as pacientes com ISC, 10 (34,5\%) foram tratadas com cirurgia radical e entre as pacientes controles, $33(29,7 \%)$. A cirurgia radical apresentou odds ratio de 1,2 (IC 95\%: 0,52-3,0, p = 0,39; teste exato de Fisher). O número de dias de utilização de dreno no pós-operatório teve mediana de 6 (2-16) entre as pacientes com ISC e 5 (1-13) nas controles ( $p=0,048$; teste de Mann-Whitney). Entre as pacientes que apresentaram ISC, 8 $(27,6 \%)$ tiveram seroma e entre as controles, 17 $(15,3 \%)$; a presença de seroma apresentou odds ratio de 2,1 (IC 95\%: 0,8-5,5, $\mathrm{p}=0,19$; teste exato de Fisher) (Tabela 2).

Tabela 2 - Fatores de risco para infecção de sítio cirúrgico (ISC) associados à terapêutica para 0 câncer de mama.

\begin{tabular}{lccc}
\hline & ISC & Controles & p \\
\hline QT neoadjuvante & $20(69,0 \%)$ & $50(45,1 \%)$ & 0,02 \\
Internação & & & \\
$\quad$ pré-operatória (dias) & $1(0-6)$ & $1(0-9)$ & ns \\
Duração da cirurgia (min) & $165(80-340)$ & $137(45-350)$ & \\
Cirurgia radical & $10(34,5 \%)$ & $33(29,7 \%)$ & ns \\
Permanência do & & & \\
$\quad$ dreno (dias) & $6(2-16)$ & $5(1-13)$ & 0,048 \\
Seroma & $8(27,6 \%)$ & $22(15,3 \%)$ & ns \\
\hline ns = não significante & & &
\end{tabular}

\section{Discussão}

O termo fator de risco tem um significado particular em epidemiologia e, no contexto da fisiopatologia e da prevenção da ISC, refere-se a uma variável que tem associação independente e significante com o desenvolvimento de ISC após cirurgia específica. Fator de risco é freqüentemente usado na literatura médica em um sentido amplo, incluindo pacientes ou aspectos da cirurgia que, embora associados com 
o desenvolvimento de ISC em análises univariadas, não são necessariamente preditores independentes. Desta forma, o estudo dos fatores de risco para o desenvolvimento de ISC em cirurgias limpas, e mais especificamente em cirurgias para o tratamento do câncer de mama, traz resultados conflitantes na literatura médica, tornando necessária a produção continuada de trabalhos que possam contribuir para esclarecimento do assunto.

A taxa de infecção pós-operatória em parede nas pacientes submetidas a cirurgia da mama, incluindo doenças benignas e malignas, varia entre $3 \%^{10}$ e $19 \%^{7}$. Uma importante razão para esta discrepância pode ser a falta de padronização no que se refere à infecção de sítio cirúrgico; alguns incluem critérios simples como a presença de pus $^{11}$, ao passo que outros referem a necessidade de diversos outros indicadores, por exemplo a classificação do CDC nos Estados Unidos da América9 ${ }^{9}$. Existem ainda definições baseadas em escores complexos $^{12}$. Nossa taxa global de infecções em sítio cirúrgico de $20,7 \%$ é comparável à encontrada na literatura, quando são utilizados os mesmos critérios de diagnóstico; Witt et al. ${ }^{13}$, em 2003, relatam taxa de $21,2 \%$ para cirurgias de tumores malignos da mama.

A identificação de fatores de risco associados às condições clínicas da paciente previamente à cirurgia é importante para buscar a compensação de eventuais desequilíbrios, tomar medidas adicionais de profilaxia ou ainda aumentar a vigilância pós-operatória naquelas pacientes consideradas de alto risco. A média de idade entre as pacientes que desenvolveram ISC foi ligeiramente maior do que a encontrada nas pacientes controles. Embora esta diferença não tenha significância estatística, alguns autores têm atribuído importância à idade como fator de risco secundário $^{13}$. A freqüência de diabete melito entre as pacientes que desenvolveram ISC foi $20,7 \%$ contra $11,7 \%$ nas pacientes controles. Esta diferença não foi estatisticamente significante, mas com base em outros estudos, nos quais a proporção pode chegar a 33,3\% dos casos, está claro que esta condição clínica aumenta o risco para infecções ${ }^{13}$.

Dos fatores de risco significantes, o estádio localmente avançado $(O R=2,7)$ e o uso de quimioterapia neoadjuvante $(O R=2,7)$ não foram passiveis de distinção em nosso estudo. Em função das características epidemiológicas do câncer de mama em nosso meio, uma proporção elevada dos casos de mama (50\%) têm sido diagnosticados como tumores localmente avançados, e nesta condição o tratamento neoadjuvante se impõe. Esta concomitância de eventos e sua elevada freqüência torna impossivel a sua separação estatística. Broadwater et al. ${ }^{4}$, sugerem que a quimioterapia neoadjuvante não aumenta as taxas de ISC quando critérios rígidos são utilizados para determinar a época exata para a realização da cirurgia, tais como contagem adequada de plaquetas e leucócitos e tumor totalmente ressecável sem a necessidade de enxerto ou cirurgia reconstrutiva. No entanto, outros autores associam a quimioterapia prévia a infecções cirúrgicas, como por exemplo em tumores da cabeça e pescoço ${ }^{14}$.

A obesidade constitui importante fator de risco evidenciado pelo IMC significativamente maior entre as pacientes que desenvolveram ISC, com média de $32,2 \mathrm{~kg} / \mathrm{m}^{2}$ versus $27,2 \mathrm{~kg} / \mathrm{m}^{2}$ nas pacientes controles. Este achado é concordante com outros estudos, que revelam risco aumentado de infecção associado à obesidade. A infecção de ferida operatória em pacientes obesas pode estar relacionada à presença do excessivo tecido gorduroso, que apresenta baixa tensão de oxigênio, o que dificultaria a cicatrização e favoreceria a ocorrência de infecção, principalmente na presença de complicações do tipo hematomas ${ }^{15}$. Também é descrita redução na responsividade de linfócitos que poderia contribuir para o aumento da taxa de infecção ${ }^{16}$. Em geral, nas pacientes obesas, tanto a tensão na linha de sutura quanto a isquemia local também são maiores do que nas não obesas. A manipulação cuidadosa dos tecidos, a hemostasia rigorosa e a técnica cirúrgica delicada podem ser fatores importantes para minimizar estes efeitos.

A duração da cirurgia está associada ao tempo de exposição da ferida operatória. Em nosso estudo, as cirurgias associadas à infecção pósoperatória tiveram duração significativamente maior que aquelas que evoluíram sem infecção. As cirurgias para o câncer de mama apresentam duração relativamente curta em comparação com outras cirurgias oncológicas, principalmente aquelas que envolvem tumores intra-abdominais. O tempo de cirurgia constitui fator de risco que é evidente principalmente quando a duração do procedimento ultrapassa quatro ho$\operatorname{ras}^{17}$.

A associação entre tempo de permanência de dreno e risco de infecção não ocorre na literatura, principalmente quando o tempo de drenagem é de cerca de quatro dias. A drenagem reduz a formação de seroma e conseqüentemente o desconforto pós-operatório e mesmo a ocorrência de infecção. Por outro lado, a permanência do dreno por um período maior não reduz a incidência de seroma $^{17}$ e, como corpo estranho, poderia facilitar 
a ocorrência de infecção. Alguns cuidados são essenciais para a utilização ótima de drenos nas cirurgias para o tratamento para o câncer de mama, podendo ser destacados a utilização de drenagem a vácuo eficiente, drenos com diâmetro adequado para um perfeito escoamento da secreção e vigilância constante para se evitarem obstruções.

As infecções em sítio cirúrgico apresentam diversos efeitos deletérios, incluindo seqüelas cosméticas e aumento do trauma psicológico associado ao tratamento do câncer de mama. Desta forma, é essencial a identificação de fatores de risco e a adoção de medidas para reduzir a sua incidência. Além dos cuidados universais recomendados pelo CDC, como a utilização de antibioticoprofilaxia e degermação de pele, deve-se buscar por meio de técnica minuciosa a redução do tempo cirúrgico. Em pacientes obesas ou com tumores localmente avançados e previamente submetidas à quimioterapia neoadjuvante deve-se intensificar a busca ativa de infecção no pós-operatório, possibilitando detectar e tratar precocemente. Os cuidados com a ferida cirúrgica, a prevenção das complicações infecciosas e o diagnóstico precoce quando estas ocorrem é parte fundamental no sucesso do tratamento do câncer de mama.

\section{ABSTRACT}

Purpose: to identify the risk factors associated with the occurrence of surgical site infection (SSI) in surgeries for the treatment of breast cancer.

Methods: the study was conducted on 140 women submitted to treatment of invasive breast cancer during the period from January 2001 to December 2002. SSI was defined as infection occurring up to 30 days after surgery and was related to the operation, according to the standard criteria adopted by the Centers for Disease Control and Prevention (CDC), USA. SSI were considered to be superficial when they involved only the skin and subcutaneous tissue and deep when they involved deep tissues at the site of incision, such as fascia and muscles. The risk factors related to patient were age, hormonal status, staging, body mass index (BMI) and hemoglobin, and the factors related to surgery were type of operation, time of hospitalization, duration of surgery, and formation of seroma and hematoma. Data concerning numerical nonparametric variables were analyzed by the Mann-Whitney test and quantitative variables were analyzed by the Fisher exact test.

Results: of the 140 patients studied, 29 (20.7\%) presented SSI, which were superficial in 19 (13.6\%) and deep in 10 (71\%); 111 patients did not present SSI and represented the control group. The risk factors associated with the patient and the disease were locally advanced stage (odds ratio $=27 ; 95 \%$ CI: 1.1-6.5) and obesity, represented by a mean BMI of $32.2 \mathrm{~kg} / \mathrm{m}^{2}$ in the patients with SSI and a mean BMI of $27.2 \mathrm{~kg} / \mathrm{m}^{2}$ in the control group ( $\left.p<0.0001\right)$. The factors related to treatment of the disease were the use of neoadjuvant chemotherapy (odds ratio $=2.7(95 \%$ CI: 1.1-6.5), the duration of surgery, whose median value was 165 minutes for the patients who developed the infection and 137 minutes for the control group $(p=0.02)$, and the number of days of use of the postoperative drain, whose median value was 6 days for the patients with SSI and 5 days for the control group $(p=0.048)$.

Conclusion: on the basis of the identification of risk factors such as advanced stage, neoadjuvant chemotherapy and obesity, preoperative care for these patients should be emphasized. The use of an accurate surgical technique may reduce the impact of other factors such as surgical time and time of use of the drain.

KEYWORDS: Surgical infections. Breast: cancer. Surgery: complications. Obesity. Diabetes mellitus.

\section{Referências}

1. Poulsen KB, Bremmelgaard A, Sorensen AI, Raahave D, Petersen JV. Estimated costs of postoperative wound infections. A case-control study of marginal hospital and social security costs. Epidemiol Infect 1994; 113:283-95.

2. Martone WJ, Jarvis WR, Culver DH, Haley RW. Incidence and nature of endemic and epidemic nosocomial infections. In: Bennett JV, Brachman PS, editors. Hospital Infections. 3rd ed. Boston: Little, Brown; 1992. p.577-96.

3. Boyce JM, Potter-Bynoe G, Dziobek L. Hospital reimbursement patterns among patients with surgical wound infections following open heart surgery. Infect Control Hosp Epidemiol 1990; 11:89-93.

4. Broadwater JR, Edwards MJ, Kuglen C, Hortobagyi GN, Ames FC, Balch CM. Mastectomy following preoperative chemotherapy. Strict operative criteria control operative morbidity. Ann Surg 1991; 213:126-9.

5. Melling AC, Ali B, Scott EM, Leaper DJ. Effects of preoperative warming on the incidence of wound infection after clean surgery: a randomised controlled trial. Lancet 2001; 358:876-80.

6. Bertin ML, Crowe J, Gordon SM. Determinants of surgical site infection after breast surgery. Am J Infect Control 1998; 26:61-5.

7. Rotstein C, Ferguson R, Cummings KM, Piedmonte MR, Lucey J, Banish A. Determinants of clean surgical wound infections for breast procedures at 
an oncology center. Infect Control Hosp Epidemiol 1992; 13:207-14.

8. Petrek JA, Peters MM, Cirrincione C, Thaler HT. A prospective randomized trial of single versus multiple drains in the axilla after lymphadenectomy. Surg Gynecol Obstet 1992; 175:405-9.

9. Mangram AJ, Horan TC, Pearson ML, Silver LC, Jarvis WR. Guideline for prevention of surgical site infection, 1999. Hospital Infection Control Practices Advisory Committee. Infect Control Hosp Epidemiol 1999; 20:250-78.

10.Read PE, Ash DV, Thorogood J, Benson EA. Short term morbidity and cosmesis following lumpectomy and radical radiotherapy for operable breast cancer. Clin Radiol 1987; 38:371-3.

11.Garner JS, Jarvis WR, Emori TG, Horan TC, Hughes JM. CDC definitions for nosocomial infections, 1988. Am J Infect Control 1988; 16:128-40.

12. Wilson AP, Webster A, Gruneberg RN, Treasure T, Sturridge MF. Repeatability of asepsis wound scoring method. Lancet 1986; 1:1208-9.
13. Witt A, Yavuz D, Walchetseder C, Strohmer H, Kubista E. Preoperative core needle biopsy as an independent risk factor for wound infection after breast surgery. Obstet Gynecol. 2003; 101:745-50.

14.Penel N, Lefebvre D, Fournier C, Sarini J, Kara A, Lefebvre JL. Risk factors for wound infection in head and neck cancer surgery: a prospective study. Head Neck 2001; 23:447-55.

15.Dindo D, Muller MK, Weber M, Clavien PA. Obesity in general elective surgery. Lancet 2003; 361:2032-5.

16.Tanaka S, Inoue S, Isoda F, et al. Impaired immunity in obesity: suppressed but reversible lymphocyte responsiveness. Int $\mathrm{J}$ Obes Relat Metab Disord 1993; 17:631-6.

17.Barber GR, Miransky J, Brown AE, et al. Direct observations of surgical wound infections at a comprehensive cancer center. Arch Surg 1995; 130:1042-7.

\title{
XI \\ Congresso \\ de \\ Ginecolo- \\ gia \\ e Obstetrícia \\ da \\ Re- \\ gião \\ Sudeste \\ da \\ FEBRASG0
}

\section{9 a 22 de maio de 2004}

\section{Belo Horizonte - MG}

\section{Informações: \\ SOGIMIG}

\author{
Tel/Fax.: \\ (31) 3222-6599 \\ e-mail: \\ sogimig@sogimig.org.br
}

